\title{
L'OCT
}

\section{en ophtalmologie}

Kate GRIEVE, Michel PAQUES, CHNO des Quinze Vingts, kategrieve@gmail.com

Depuis l'invention de l'ophtalmoscope en 1850 par Helmholtz, le domaine de l'imagerie de la rétine a connu encore une grande avancée en 1991 avec l'invention de la tomographie par cohérence optique (optical coherence tomography, OCT) par James Fujimoto au MIT. Cette technique interférométrique a introduit pour la première fois dans l'histoire de l'imagerie de l'œil la possibilité d'observer les couches cellulaires de la rétine en coupe virtuelle en profondeur de manière non invasive. Depuis cette date l'OCT a continuellement évolué et son champ d'application ne cesse de se développer au point d'être devenu l'examen de première intention dans le domaine des maladies rétiniennes.

\section{[}

a tomographie par cohérence optique est devenue un standard de soins en ophtalmologie, fournissant des informations en temps réel sur la structure et la fonction - diagnostiquer la maladie, évaluer la progression et la réponse au traitement, tout en aidant à comprendre la pathogenèse de la maladie et à créer de nouveaux traitements.

Grâce à l'invention de l'OCT, avec un contraste provenant des différences d'indice de réfraction des tissus, l'ophtalmologue a eu accès, pour la première fois, à l'image des différentes couches de la rétine, en temps réel, in vivo. Alors que la rétine du fait de sa transparence reste invisible aux techniques traditionnelles d'imagerie du fond d'œil, elle est, avec l'OCT, devenue visible, à une échelle de résolution micrométrique qui permet de déceler les modifications pathologiques de façon précoce. La visualisation de la rétine en coupe est rapidement devenue une routine quotidienne de l'ophtalmologiste, et le développement de l'OCT vers un dispositif commercial a progressé à grande vitesse. En effet, il ne s'est écoulé que cinq ans entre la première démonstration de la technique en milieu de recherche jusqu'à son arrivée sur le marché. Des améliorations techniques successives ont permis de gagner en vitesse d' acquisition et en résolution, et les traitements d'images automatisés ont permis une diversification de structures accessibles (la rétine mais aussi la choroïde, la cornée) permettant à l'OCT de devenir la technique de référence en ophtalmologie. Cette technique est maintenant appliquée à d'autres domaines d'imagerie tels que la dermatologie, la cardiologie, la cancérologie, etc. Le succès de l'OCT a aussi été obtenu grâce aux ophtalmologues, qui sont habitués à l'adoption et au développement de nouvelles technologies d'imagerie, et à son excellente tolérance, étant un examen rapide et non éblouissant.

Les ventes de machines OCT ont évolué exponentiellement depuis 2002, avec un marché actuel de plus d'un milliard d'euros par année. À l'échelle mondiale, on estime à plus d'un million le nombre d'examens OCT pratiqués chaque jour, et à 20000 le nombre de personnes employées par la filière.

Nous ferons ici le point sur la chronologie des systèmes OCT développés pour l'ophtalmologie (domaine temporel, domaine spectral, source
SUPPLY

\section{HIGH HARMONICS \\ GENERATION SOURCES}

AND BEAMLINES

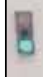

\section{HHG BEAMLINE FULLY OPERATIONAL SINCE 2018} AT ELI-BEAMLINES
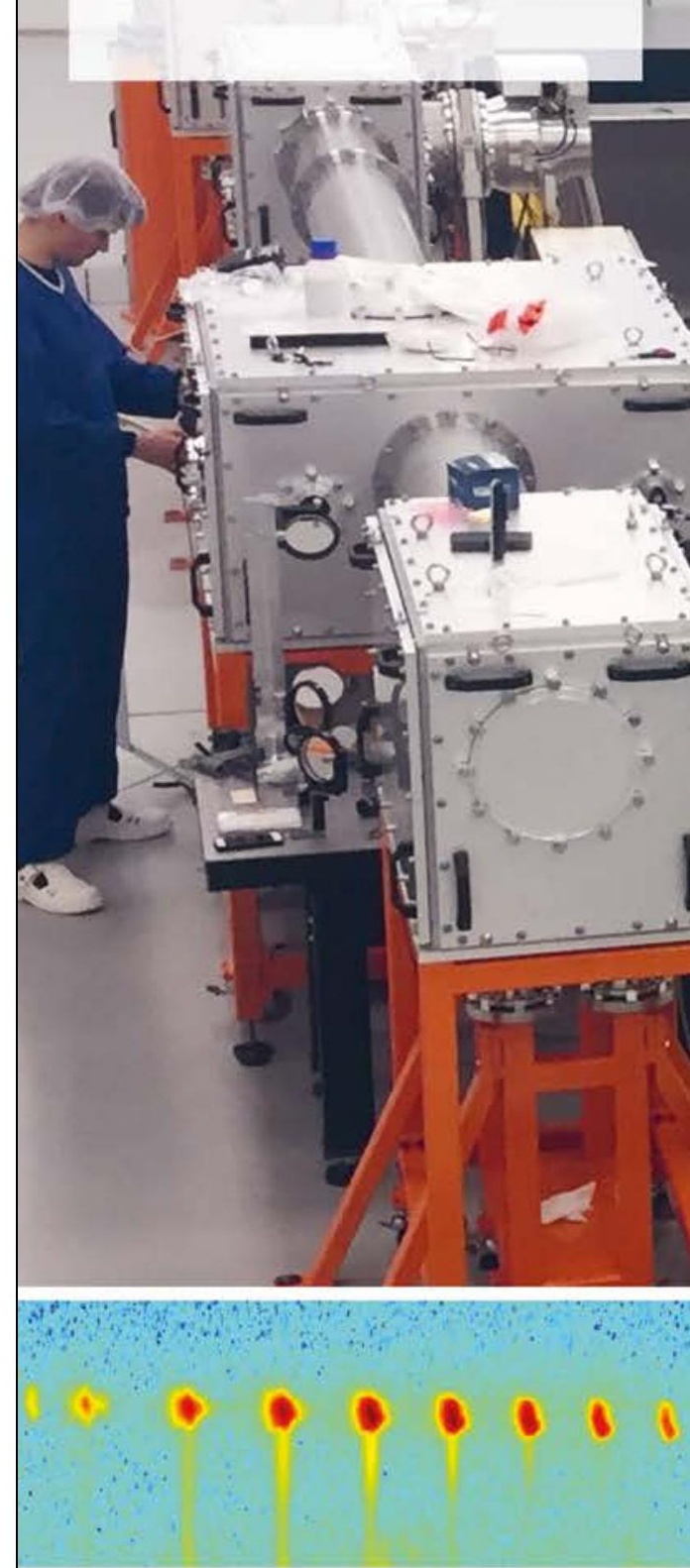
Domaine temporel

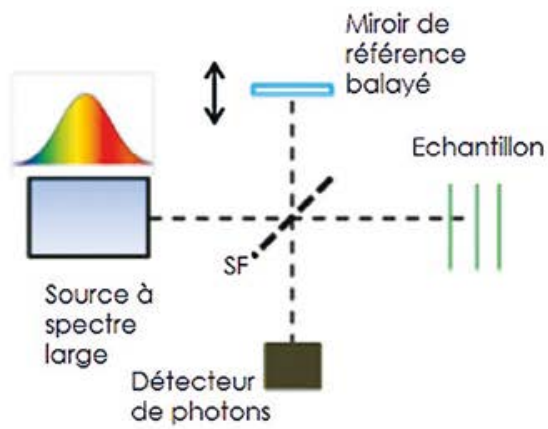

à spectromètre

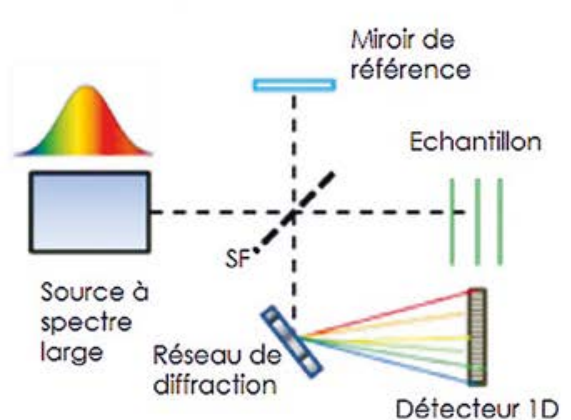

à source balayée

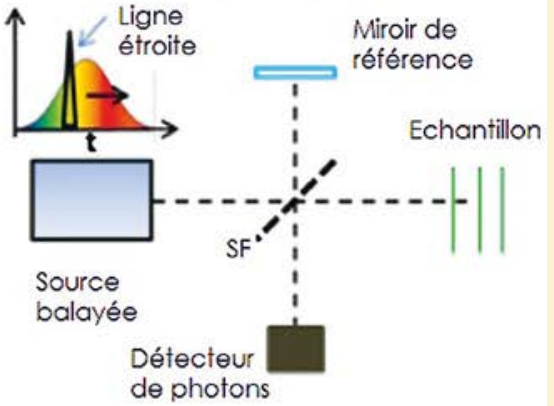

Figure 1. Schéma du principe de l'OCT en domaine temporel et spectral ; SF, séparateur de faisceau. Image modifiée de Drexler et al., Optical coherence tomography today: speed, contrast, and multimodality, J. of Biomedical Optics, 19(7), 071412 (2014). https://doi.org/10.1117/1. JBO.19.7.071412

balayée) avec un bref rappel technique $\mathrm{du}$ fonctionnement de ces systèmes. Ensuite nous présenterons l'application de l'OCT en ophtalmologie à l'imagerie de la rétine et de la cornée, en exposant des cas cliniques des principales pathologies oculaires qui sont les causes majeures de cécité en France. Nous évoquerons aussi les nouveautés qui permettent une imagerie fonctionnelle de la rétine, par exemple l'OCT angiographie (OCT-A). Pour terminer, nous discuterons des perspectives.

\section{Historique technique}

L'OCT peutêtre considéré commel'analogue optique de l'échographie, où l'on cherche à mesurer le temps d'écho d'un faisceau de lumière infrarouge. Comme la lumière se déplace beaucoup plus vite que le son (d'un facteur $10^{6}$ ) il faut avoir recours à une méthode de mesure indirecte, l'interférométrie. Le montage OCT, basé sur l'interféromètre de Michelson, se sert de la propriété de cohérence de la source d'illumination

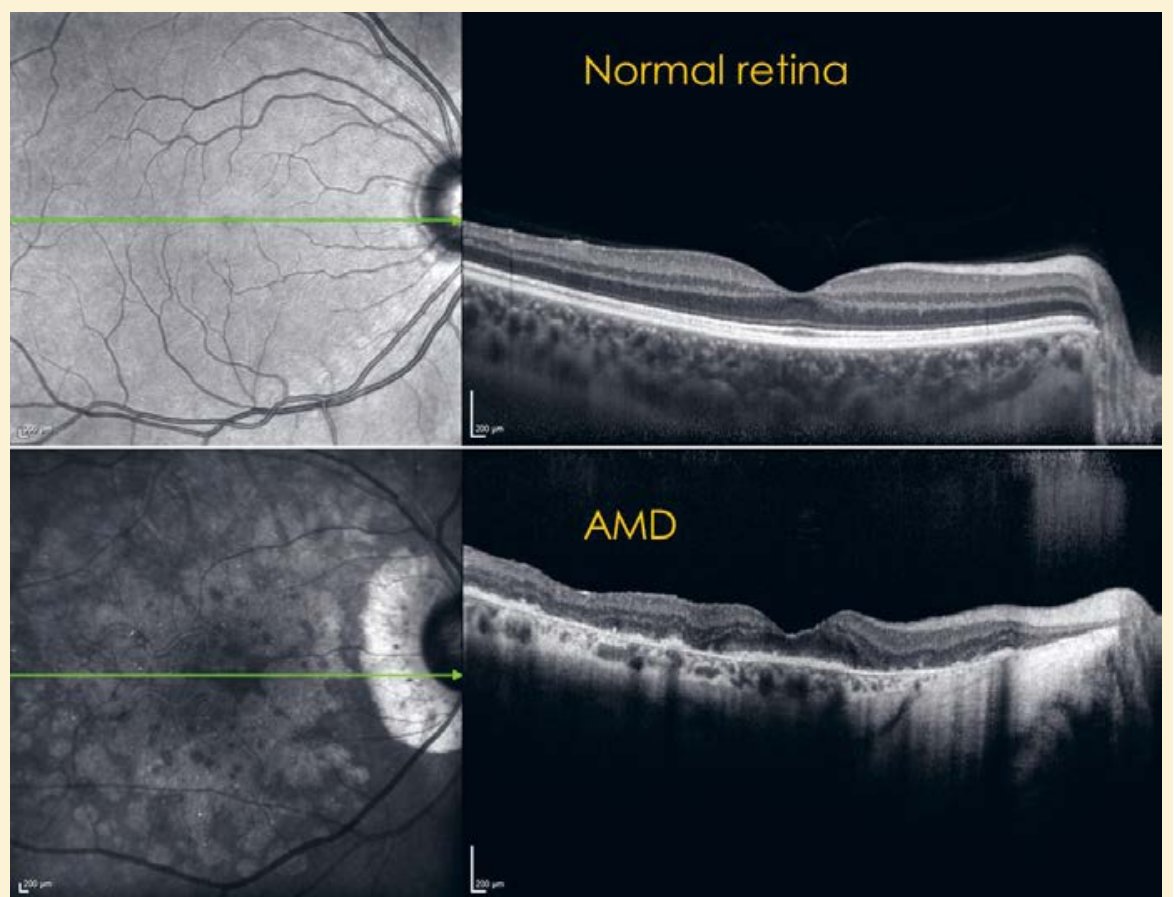

Figure 2. Des images prises avec le «Spectralis» de Heidelberg montrent la vue de face par ophtalmoscope laser à balayage, et la vue en coupe par OCT. Rétine normale (haut) et atteinte de dégénérescence maculaire liée à l'âge (DMLA) (bas) : cas avancé de DMLA sèche avec atrophie géographique; notez l'absence des couches de photorécepteurs dans la zone centrale. (Image CHNO des XV-XX) pour produire des interférences permettant de localiser une couche précise de l'échantillon, si une source de spectre large est employée. En effet, l'interféromètre corrèle les signaux de ses deux bras: la lumière rétrodiffusée de l'échantillon interfere avec la lumière réfléchie d'un miroir de référence avec un temps de parcours connu. Les interférences ne sont observées que quand les longueurs de chemin optique entre le bras échantillon et le bras de référence sont égales, à la longueur de cohérence près: si la longueur de cohérence est faible (spectre large), on obtient une meilleure précision axiale. La présence des interférences sert comme mesure relative de la distance parcourue par la lumière, et permet donc une séparation des échos optiques, surtout dans un échantillon avec plusieurs surfaces réfléchissantes peu espacées, comme la rétine.

L'OCT en domaine temporel (time domain) balaye la position de son miroir de référence selon l'axe $z$ pour générer une ligne axiale dans la profondeur du tissu (A-scan). Ensuite, un balayage latéral permet de reconstituer une image en coupe (B-scan), à partir d'une série de A-scans successifs à différentes positions latérales de l'échantillon. Une pile de données tridimensionnelle peut être générée à partir d'une série de coupes, en balayant le faisceau incident en trame. L'OCT en domaine de Fourier, ou domaine spectral, en revanche, garde une position de miroir de référence fixe (donc sans balayage axial) pour détecter tous les échos de lumière de façon 


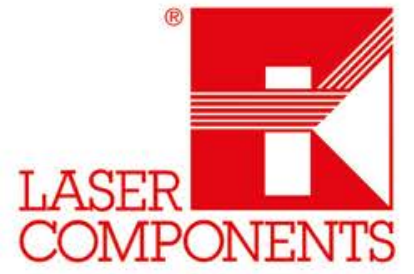

gueur d'onde ou de leur fréquence. Les mélanges spectraux d'interférence dans chaque A-scan sont séparés par une analyse spectrale des longueurs d'onde par transformée de Fourier. Un balayage latéral est toujours effectué pour acquérir une séquence de A-scans pour reconstruire les coupes et volumes. La cadence de variation d'intensité aux différentes fréquences indique la localisation des différentes couches réfléchissantes de l'échantillon. L'interférogramme résultant varie en fréquence selon la différence de chemin optique entre les deux bras. Plus grande est la différence de chemin optique, plus grande est la fréquence résultante, donc il y a un codage en fréquence de la profondeur. On peut ainsi extraire l'information sur la fréquence et l'amplitude du signal grâce à une transformée de Fourier, et obtenir le A-scan en entier d'un coup.

L'OCT en domaine spectral est effectué soit en remplaçant le détecteur simple par un spectromètre - on place un prisme ou une grille devant le détecteur pour étaler la lumière en spectre; soit en remplaçant la source lumineuse par une source balayée en longueur d'onde (swept source), c'est-à-dire un laser de longueur d'onde variable pour balayer le spectre de façon séquentielle. L'avantage de celui-ci

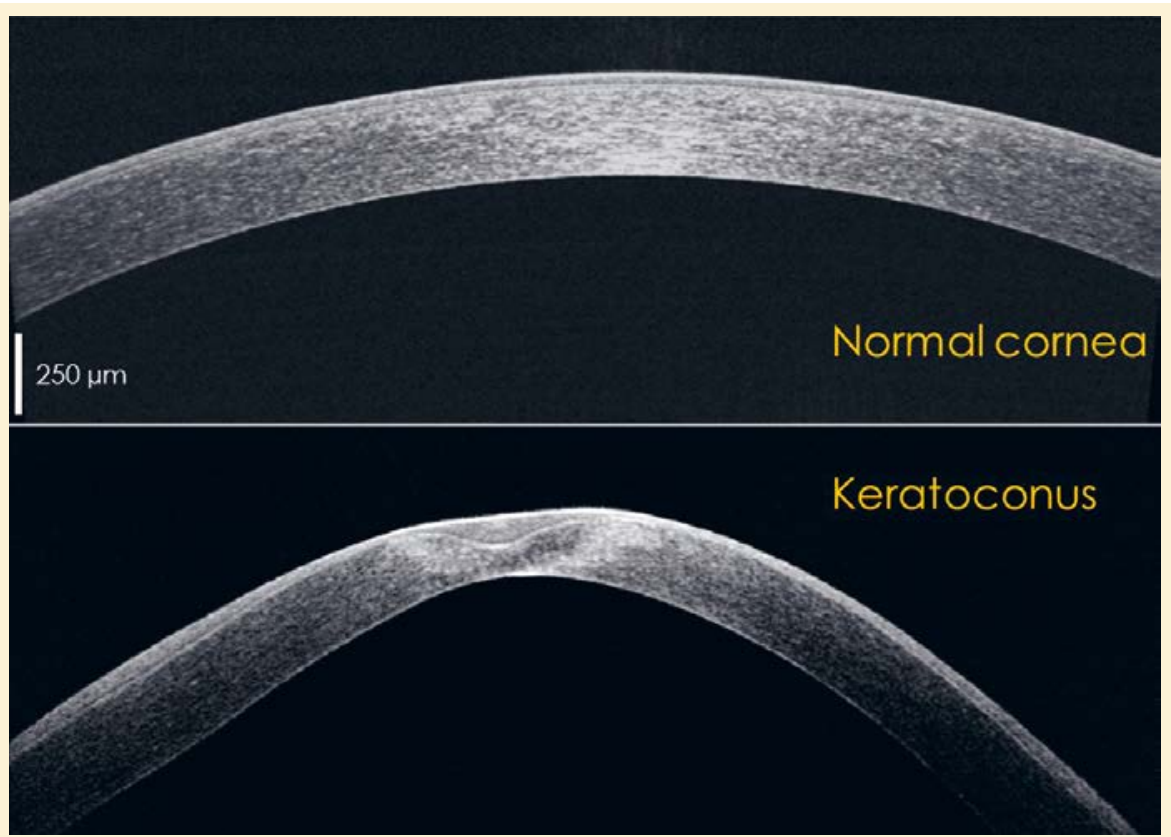

Figure 3. Images prises avec l'OCT « Optovue ». Cornée normale (haut) et atteinte de kératocône (bas) : notez la forme conique, l'amincissement du stroma et l'épaississement de l'épithélium. (Image CHNO des XV-XX) est qu'on n'est plus limité par la résolution du spectromètre, et donc on peut atteindre une plus grande profondeur de pénétration dans les tissus (jusqu'à la choroïde dans le cas de l'œil). L'OCT en domaine temporel capture l'image pixel par pixel (balayage axial et latéral) tandis que l'OCT en domaine spectral capture l'image ligne par ligne et n'a besoin que d'un balayage latéral. L'OCT en domaine spectral a donc un avantage de rapidité, avec un meilleur rapport signal sur bruit, par rapport à l'OCT en domaine temporel, et souffre moins d'artefacts liés au mouvement de l'œil. L'OCT en domaine spectral est le standard actuel en clinique pour les systèmes ophtalmiques, et acquiert à des vitesses de 25000 à $80000 \mathrm{~A}$-scans/seconde.

\section{OCT en ophtalmologie : imagerie de la rétine}

L'observation de la rétine par OCT a permis une révolution de la prise en charge principalement de deux maladies de la rétine : l'œdème maculaire diabétique et la dégénérescence maculaire liée à l'âge (DMLA). Dans ces deux malacar les modifications de l'épaisseur et/ ou de la structure rétinienne sont hautement prédictives de la survenue de dies, il permet de guider le traitement,

\section{small components MASSIVE IMPACT}

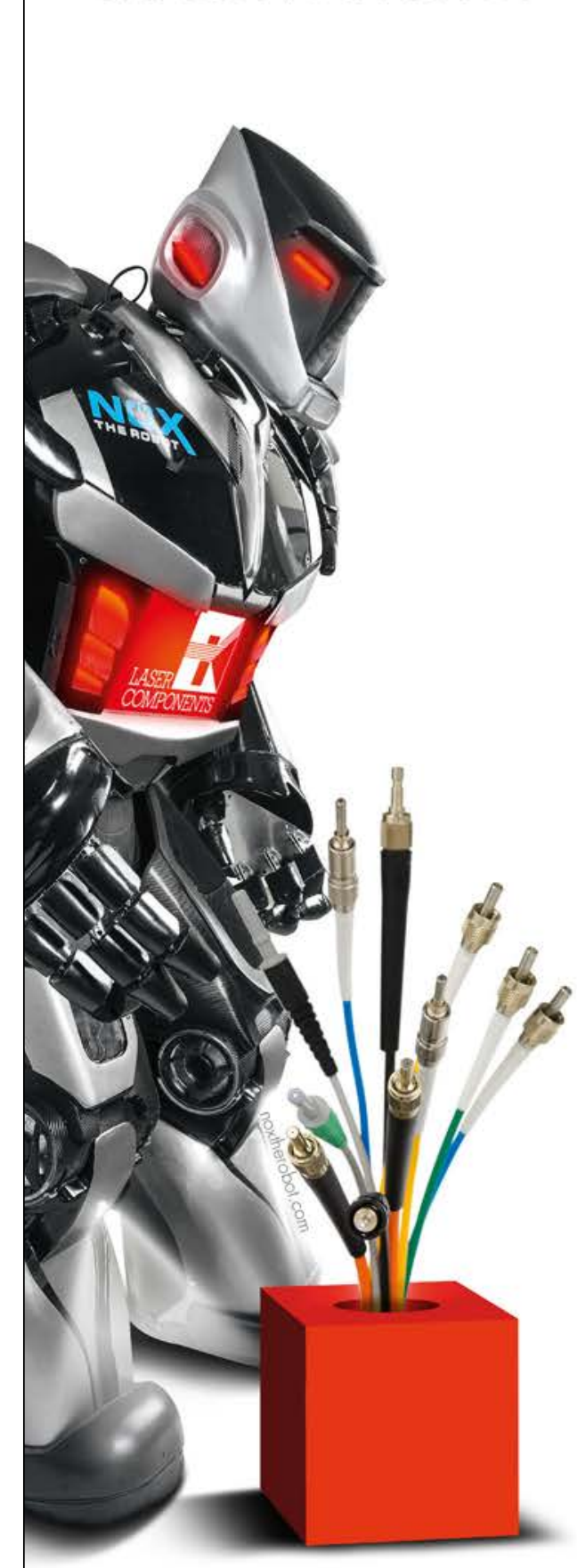




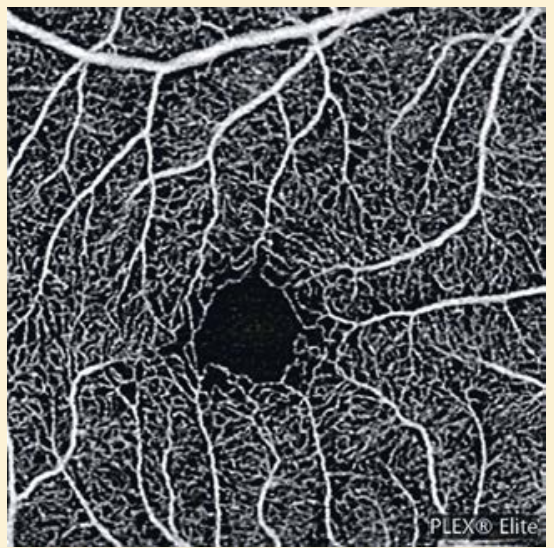

Figure 4. OCT-A d'un sujet sain : le mouvement des globules dans les vaisseaux révèle le réseau de vaisseaux rétiniens. (Image CHNO des XV-XX)

complications visuelles. En pratique, des comparaisons automatiques d'examens successifs permettent de détecter aisément une modification de l'épaisseur de la rétine, localisée ou généralisée ; en principe, une augmentation de l'épaisseur rétinienne traduit une récidive d'activité de la maladie et peut faire décider à elle seule de la reprise du traitement. Un traitement préventif (avant la survenue de baisse visuelle) est donc possible. Ceci permet d'optimiser l'administration des soins. C'est en particulier la démonstration de l'intérêt de l'OCT dans la DMLA qui a été le véritable moteur du succès de l'OCT.

L'OCT est donc devenu l'examen de référence pour la mesure de l'épaisseur rétinienne ainsi que de sa structure; il est en effet possible de documenter la perte d'un seul type de cellules, voire d'un seul type de structures cellulaires comme les segments externes des photorécepteurs. Il est même possible d'estimer la disposition spatiale des photorécepteurs (OCT directionnel). L'OCT a permis également de découvrir d'autres biomarqueurs comme l'épaisseur de la choroïde, modifiée dans certaines maladies affectant la vision.

Des systèmes d'OCT sont également installés sur des microscopes opératoires pour aider le chirurgien lors d'interventions sur la rétine. Des systèmes destinés à un usage pédiatrique voire néonatal ont également été développés.

\section{Cornée saine et pathologique}

Bien que l'essentiel de l'utilisation de l'OCT en ophtalmologie concerne la rétine, la cornée, transparente tout comme la rétine, est aussi l'objet d'examens par OCT. Ce dernier permet d'observer les différentes couches de la cornée : épithélium, stroma, endothélium, et de mesurer certains paramètres comme la profondeur de la chambre antérieure (espace entre la cornée et l'iris). Il est utile pour mesurer l'épaisseur de la cornée avant chirurgie réfractive, et aussi pour diagnostiquer et suivre certaines pathologies comme le kératocône (déformation conique et amincissement progressif de la cornée).

\section{Imagerie fonctionnelle pari OCT - angiographie}

L'angiographie par OCT (OCT-A) révèle les vaisseaux rétiniens grâce à un traitement d'images qui mesure la variance temporelle à court terme du signal, due à la circulation des globules rouges. L'OCT-A ne nécessite pas d'agent de contraste et est donc non invasive. À l'heure actuelle, il reste néanmoins qualitatif et non quantitatifen raison du faible échantillonnage. Malgré ses limites, l'OCT-A a permis de relancer l'intérêt pour l'étude de la circulation oculaire. Ceci a permis de mieux comprendre, et bientôt de mieux

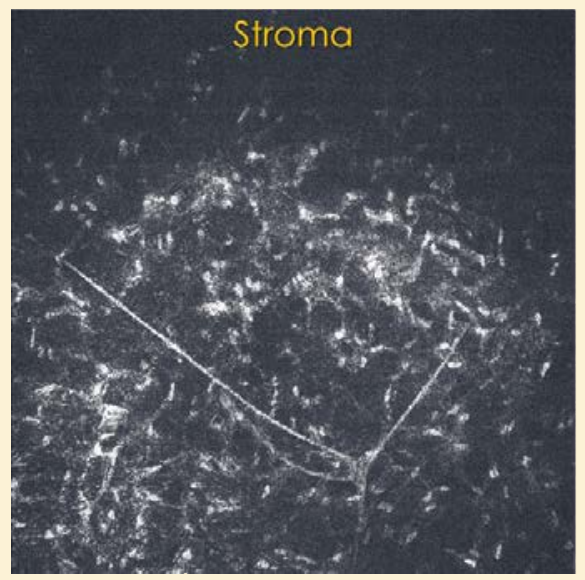

Figure 5. Images prises avec un prototype de laboratoire par OCT plein champ in vivo. Cornée de sujet sain : deux plans dans l'orientation de face révèlent un nerf et les cellules individuelles dans le stroma (gauche) et l'endothélium (droite, cellules endothéliales hexagonales indiquées par flèches). (Image V. Mazlin, Institut Langevin)

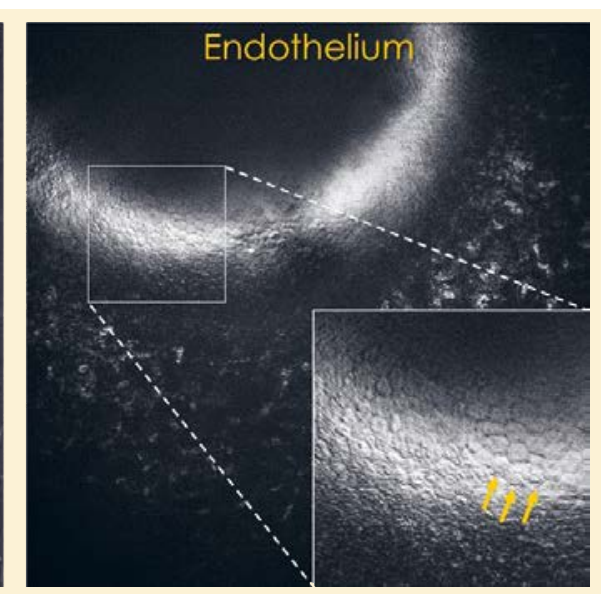

suivre, les patients atteints de DMLA car il permet de détecter les néovaisseaux responsables de la perte visuelle. De même, l'OCT Doppler permet de quantifier la vitesse du flux sanguin dans les vaisseaux, avec la limite inhérente à toute mesure unidirectionnelle.

\section{Perspectives}

L'OCT est une des technologies médicales s'étant le plus rapidement transférées du laboratoire vers la clinique et, depuis deux décennies, les applications ne cessent des'étendre, s' approchantau plus près d'une microscopie cellulaire in vivo. Des évolutions futures devraient encore permettre d'en améliorer la robustesse et la précision, ainsi que son association avec d'autres modalités d'imagerie ou de fonction, renforçant son rôle d'examen de référence en ophtalmologie.

Pour avancer vers une (encore) meilleure résolution, l'OCT peut ainsi être couplé à un système d'optique adaptative (AO-OCT). L'optique adaptative permet de mesurer et corriger en boucle fermée et en temps réel les distorsions du front d'onde, et donc d'améliorer la résolution spatiale de l'OCT et augmenter le rapport signal sur bruit. L'AOOCT permet d'atteindre un niveau de détail à une résolution cellulaire dans la rétine, avec des volumes de données tridimensionnelles à partir desquelles on peut extraire des coupes dans les plans des capillaires, des fibres, et des photorécepteurs [2]. On peut même 
combiner l'AO-OCT avec le contraste du mouvement (motion contrast) à très petite échelle pour révéler les cellules transparentes telles que les cellules ganglionnaires ou de l'épithélium pigmentaire [3]. Cependant, ces systèmes restent complexes et onéreux, et sont essentiellement adaptés à la recherche.

Une autre méthode pour atteindre une résolution cellulaire dans les tissus oculaires in vivo est l'OCT plein champ, une technologie française qui permet de faire de l'imagerie plan par plan dans l'orientation en face, et qui exploite la propriété d'incohérence spatiale de sa source, en s'appuyant sur un interféromètre de type Linnik doté d'un objectif de microscope sur chaque bras, pour obtenir des images de cornée et de rétine in vivo à résolution micrométrique [4] sans optique adaptative.

Enfin, l'intelligence artificielle trouve ici un terrain très riche pour mettre au point des systèmes d'analyse automatisée des images. Il est très probable que des systèmes d'aide au diagnostic, voire d'aide à l'acquisition des images seront rapidement disponibles sur les systèmes OCT.

\section{Conclusion}

L'histoire de l'OCT est un cas exemplaire de transfert rapide du laboratoire vers les soins quotidiens. Ce processus a permis à Fujimoto d'introduire le concept d'un «écosystème » comportant recherche, financement public, études cliniques, innovation, entrepreneuriat et industrie, qui doivent tous fonctionner en synergie [5]. Ce processus est difficile, mais doit inspirer les professionnels dans le domaine biomédical pour un transfert d'innovation le plus rapide et le plus efficace possible.

\section{POUR EN SAVOIR PLUS}

[1] Drexler W., Fujimoto J.G., Optical coherence tomography: technology and applications

$2^{\text {nd }}$ edn. Springer Science \& Business Media (2015)

[2] Jonnal R.S., Kocaoglu O.P., Zawadzki R.J., Liu Z., Miller D.T., Werner J.S., A Review of Adaptive Optics Optical Coherence Tomography: Technical Advances, Scientific Applications, and the Future, Invest. Ophthalmol. Vis. Sci. 57(9), OCT51-OCT68 (2016)

https://doi.org/10.1167/iovs.16-1910

[3] Liu Z., Kocaoglu O.P., Miller D.T., 3d imaging of retinal pigment epithelial cells in the living human retina, Invest. Ophthalmol. Vis. Sci. 57(9), OCT533 (2016) https://doi.org/10.1167/iovs.16-19106

[4] Mazlin V., Xiao P., Dalimier E., Grieve K., Irsch K., Sahel J.A., Fink M., Boccara A.C., In vivo high resolution human corneal imaging using full-field OCT, Biomed. Opt. Express 8(2), 557-568 (2018)

[5] Fujimoto J., Swanson E., The development, commercialization, and impact of optical coherence tomography. Invest. Ophthalmol. Vis. Sci. 57(9), OCT1-OCT13 (2016) https://doi.org/10.1167/iovs.16-19963

\section{Découvrez l'Annuaire des formations en optique photonique}

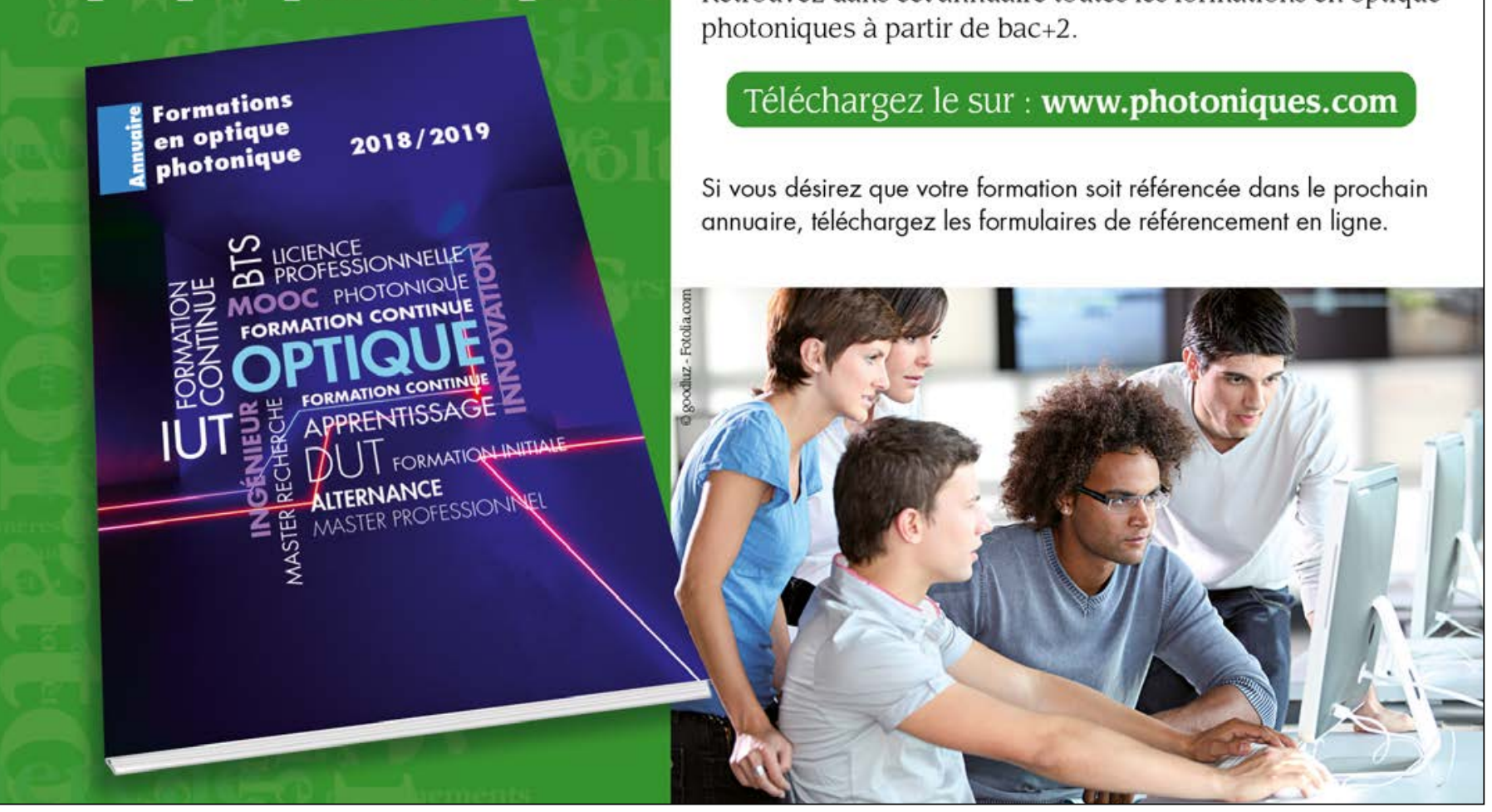

L'annuaire des formations en optique photonique est destiné aux responsables RH des entreprises et aux futurs étudiants.

Retrouvez dans cet annuaire toutes les formations en optique photoniques à partir de $\mathrm{bac}+2$.

\section{Téléchargez le sur : www.photoniques.com}

i vous désirez que votre formation soit référencée dans le prochain 